\section{Efficacy of physical therapy associated with botulinum toxin type $A$ on functional performance in post-stroke spasticity: A randomized, double-blinded, placebo-controlled trial}

\author{
Antonio Prazeres, Marília Lira, \\ Paula Aguiar, Larissa Monteiro, Ítalo \\ Vilasbôas, Ailton Melo \\ Division of Neurology and \\ Epidemiology, Federal University \\ of Bahia, Salvador-Bahia, Brazil
}

\begin{abstract}
The aim was to investigate if botulinum toxin type A (BTx-A) associated with physical therapy is superior to physical therapy alone in post stroke spasticity. A randomized, double-blinded controlled trial was performed in a rehabilitation unit on Northeastern, Brazil. Patients with post stroke spasticity were enrolled either to BTx-A injections and a pre-defined program of physical therapy or saline injections plus physical therapy. Primary endpoint was functional performance evaluated through time up and go test, six minutes walking test and Fugl-Meyer scale for upper limb. Secondary endpoint was spasticity improvement. Confidence interval was considered at $95 \%$. Although there was a significant decrease in upper limbs flexor tonus $(\mathrm{P}<0.05)$ in the BTx-A group, there was no difference regarding functional performance after 9 months of treatment. When analyzing gait speed and performance, both groups showed a significant improvement in the third month of treatment, however it was not sustained over time. Although BTx-A shows superiority to improve muscle tone, physical therapy is the cornerstone to improve function in the upper limbs of post stroke patients.
\end{abstract}

\section{Introduction}

Stroke is the major cause of permanent and temporary functional incapacity worldwide among adults, affecting limb strength, motor control, balance and mobility. ${ }^{1}$ Spasticity is characterized by an increase in tonic stretch reflex movement velocity dependent and post-stroke spasticity is frequently associated with poor functional performance due to abnormal postural patterns, leading to retractions, atrophy, selective movement control loss, limb weakness, fibrosis and structured contractions. ${ }^{2}$ Moreover, impairment in activities of daily living (ADL) such as feeding, locomotion, proper hygiene and sleeping habits results in poor quality of life (QOL) and increased burden to relatives and caregivers. ${ }^{3}$

Several trials support the efficacy and safety of botulinum toxin type A (BTx-A) on spasticity treatment, reducing muscle permanent contraction and abnormal postural patterns, therefore, favoring rehabilitation process. ${ }^{4}$ Physical therapy has been described to be effective in post-stroke spastic patients through prevention of secondary incapacities and promoting behavioral reeducation, based on biomechanical and neurophysiological patterns. These techniques include physical exercises that focus on functional rehabilitation, reduction of limb spasticity, muscle strength improvement and sustained joint movement amplitude, besides proprioceptive and sensorial stimuli. 5

Several trials with BTx-A show functional improvement in post-stroke spastic patients when compared to placebo, however, none have studied the impact of physical therapy alone. 4

The aim of this trial was to investigate if BTx-A treatment associated with physical therapy is superior to physical therapy alone on functional performance in post-stroke spastic patients.

\section{Materials and Methods}

\section{Population}

A randomized, double-blinded, placebo-controlled trial was conducted with poststroke subjects attending a neurorehabilitation unit at an University Hospital in Northeastern, Brazil, between August 2009 and September 2012.

Inclusion criteria were defined diagnosis of post-stroke spasticity, age between 50-70 years-old, being in a regular program of physical therapy, at least one-year and no more than five-year history between the vascular event and study inclusion. Subjects who presented with conditions that impaired research procedures such as uncontrolled hypertension, structured joint contractions, prior BTx-A treatment in the last six months, regular use of medications to spasticity, renal or hepatic chronic diseases, hematological disorders, and pregnant or breast-feeding women were excluded (Figure 1).
Correspondence: Ailton de Souza Melo, Rua Padre Feijo, S/N, Canela, CPPHO, Quarto andar, Salvador-Bahia, Brazil.

Tel.: +55.71999755275

E-mail: asm@ufba.br

Key words: Botulinum toxin; Physical therapy; Stroke; Spasticity; Clinical trial.

Contributions: the authors contributed equally.

Conflict of interest: the authors declare no potential conflict of interest.

Funding: This work was funded by Brazilian National Institutes of Science (CITECS/INNT/CNPq), CAPES, and UFBA.

Received for publication: 27 August 2017.

Revision received: 8 December 2017.

Accepted for publication: 22 January 2018.

This work is licensed under a Creative Commons Attribution NonCommercial 4.0 License (CC BY-NC 4.0).

CCopyright A. Prazeres et al., 2018

Licensee PAGEPress, Italy

Neurology International 2018; 10:7385

doi:10.4081/ni.2018.7385

\section{Randomization}

Eligible subjects were allocated into two intervention groups through a randomized block system (blocks of ten) with codified numbers drawn from an opaque envelope. The files that identified the group allocation were archived and investigators and patients remained blinded during all study procedures.

\section{Study procedures}

All patients were oriented previously about study procedures and evaluated by a specialized physical therapist. In the first room, socio-demographic questionnaires were applied, and tonus was evaluated with the modified Ashworth scale (MAS). All physical measures were performed with the patient positioned in dorsal decubitus.

Muscles selected to BTx-A injections should present with a degree of hypertonia equal or superior to two.

In a second room, all patients were evaluated for their functional performance through the time up and go test (TUG), six minutes walking test and Fugl-Meyer scale to upper limb, applied with the patient seated comfortably in a chair with backrest. The TUG test measures the time spent to raise from a chair, walk 3 meters, take a 180degree turn and come back to the chair with a normal value of 10 seconds or less. The six minutes walking test measures the dis- 
tance covered by the patient in this time interval.

In sequence, patients were placed in a third room were BTx-A injections were applied by a specialized neurologist on predetermined muscles. A nurse prepared injections with their respective codes, derived from randomization. Patients allocated to intervention group received BTx-A

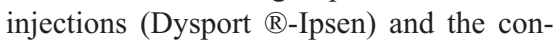
trol group received injections prepared with saline solution. Patients and injectors remained blinded regarding the intervention. Since the baseline evaluation all patients were included in a pre-determined protocol of physical exercises including muscle strength, flexibility, endurance and functional training. Sessions were scheduled twice a week, with an interval between sessions of 24h. Each session lasted $30 \mathrm{~min}-$ utes with one-minute break between each activity involving physical effort. The first five minutes of each session consisted of flexibility activities with sustained stretching (15 seconds) and joint mobilization on the affected limb, followed by muscle strength training with concentric and eccentric movements with progressive loads depending on the patient performance in the following 10 minutes. The final movements consisted of gait and upper limb functional training combined with endurance exercises. These activities were performed in two different days: trunk, upper limb and arm functional training in the first day and pelvis, lower limb and gait training in the second day. The same instructor previously trained all physical therapists before performing study procedures.

Data were collected and placed in a sealed envelope. Evaluations and injections were performed at baseline, 3 and 6 months from the first intervention, and a last evaluation was performed within 9 months (Figure 2).

\section{Outcomes}

Primary endpoint was functional performance evaluated through TUG test, six minutes walking test and Fugl-Meyer scale for upper limb. Secondary endpoint was spasticity improvement, and only a decrease of at least one point in modified Ashworth scale was considered as clinically relevant.

\section{Ethics}

All subjects included previously signed an informed consent term and study protocol was approved by the local ethics committee under the number 064/2009 and CEP. 059/09.

\section{Statistical analysis}

For descriptive analysis we used to cal- culate frequencies for qualitative variables and measures of central tendency and dispersion of quantitative variables. In relation to the instruments of evaluation (FuglMeyer, Time Up and Go, six minutes walking test) the scores obtained were compared to the treatment follow-up time (baseline, 3 months, 6 months and 9 months). For this, repeated measures ANOVA were per- formed, and when necessary post-hoc test with Fisher LSD. For analysis of muscle tone, a non-parametric Friedman analysis was performed. This statistical test was used to compare the difference between the degrees of spasticity in the pre-injection of TB-A (baseline) and the follow-up time of 3-6-9 months. The level of significance adopted was $\mathrm{P}<0.05$.

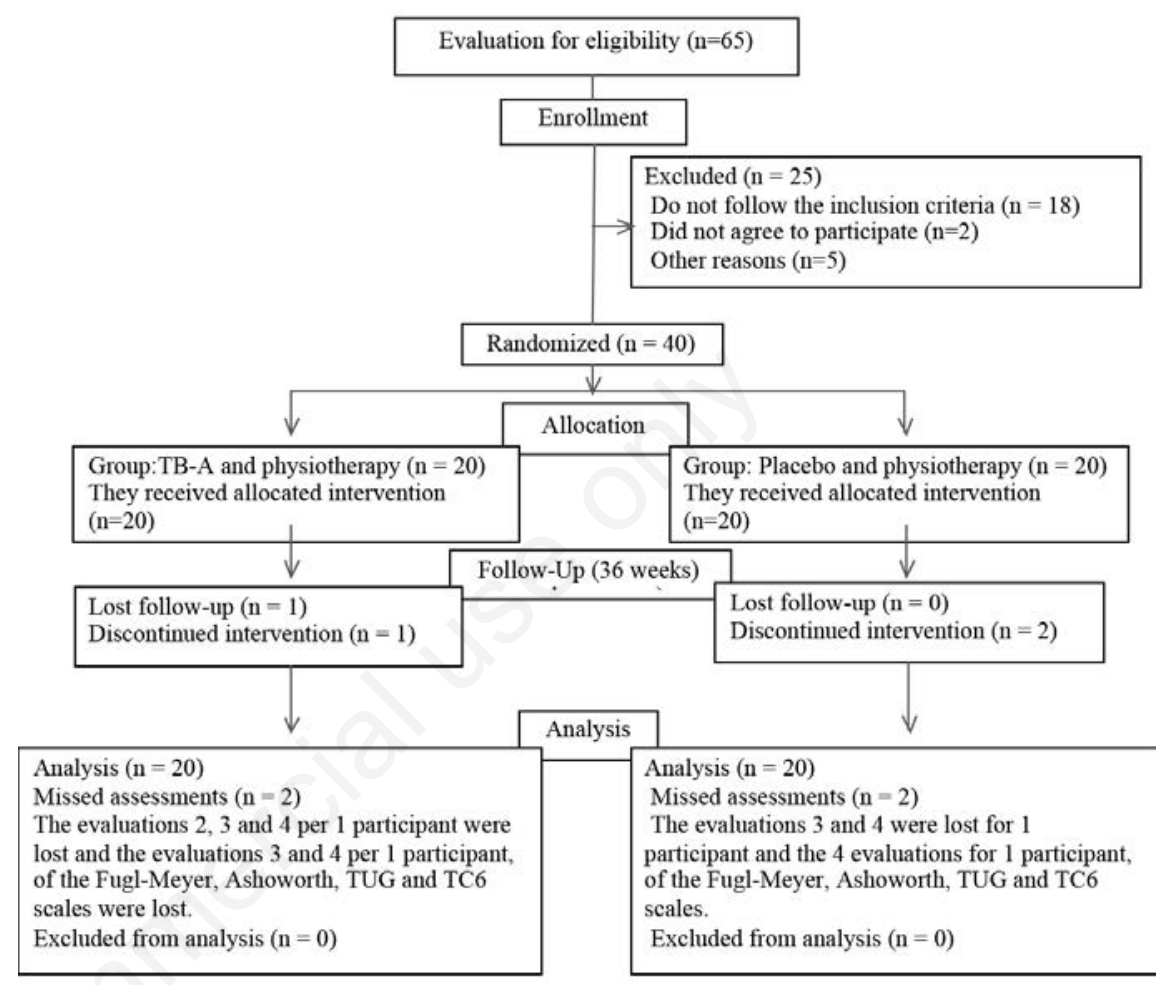

Figure 1. Recruitment process, randomization, allocation, follow-up and analysis regarding the participants of the groups.

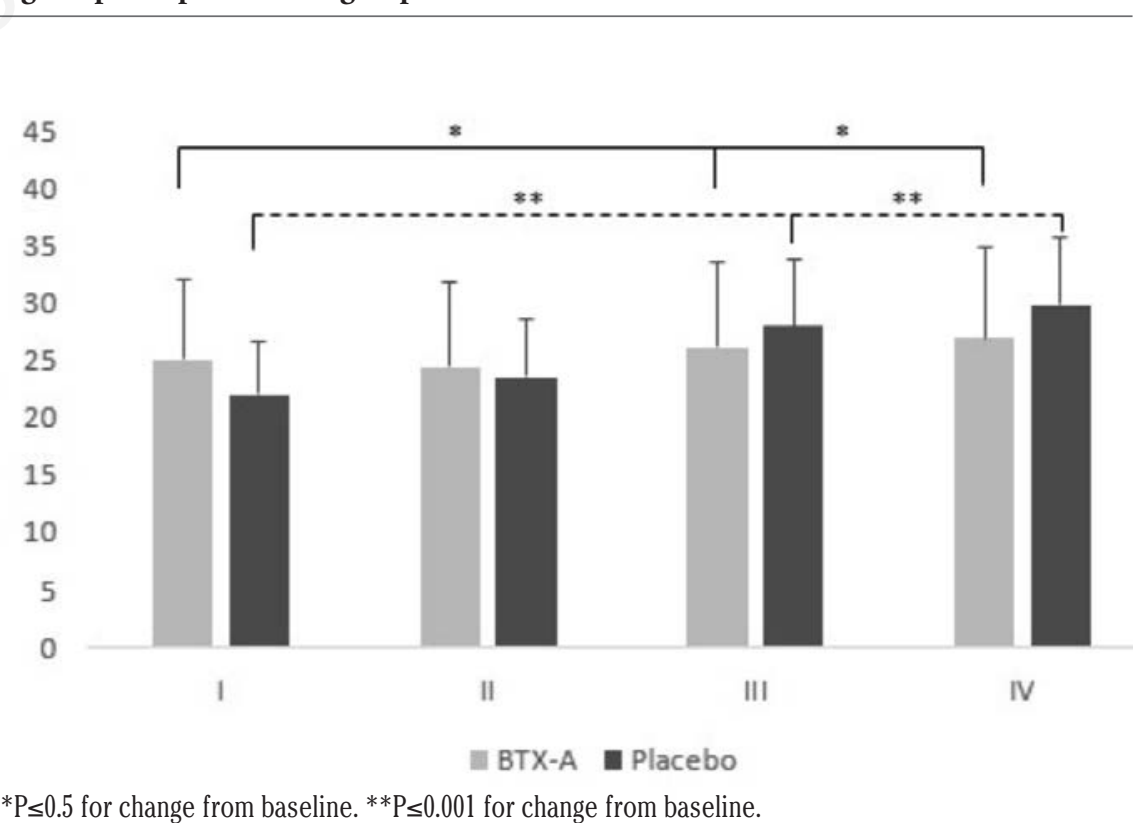

Figure 2. Changes during the follow-up time in the Total score Fugl-Meyer Scale. 


\section{Results}

Initially forty patients were randomized to btx-A and placebo groups, however, three did not completed study procedures. Demographic characteristics of the subjects that completed study procedures are described in Table 1. Baseline data and clinical measures performed at each study visit are presented in Table 2 .

\section{Upper limb function evaluation}

Upper limb motor function measured through Fugl-Meyer scale showed a significant increase after interventions that remained consisted during trial length in both groups, when analyzing the total score $\left(\mathrm{F}_{3,102}=17 \quad \mathrm{P}>0.001, \mathrm{j}^{2}=0.33\right)$. A greater improvement was seen after six months from the first intervention $(\mathrm{P}<0.001)$. When comparing groups, there was a low interaction among them during the follow-up period $\left(\mathrm{F}_{3,102}=2.7, \mathrm{P}=0.046, \mathrm{y}^{2}=0.07\right)$.

In both groups, we observed significant improvement after six months of treatment in the following Fugl-Meyer domains: global evaluation $\left(\mathrm{F}_{3,102}=2.7, \quad \mathrm{P}=0.046\right.$, $\left.\eta^{2}=0.07\right)$, wrist stability $\quad\left(F_{3,102}=3.04\right.$ $\left.\mathrm{p}>0.032, \eta^{2}=0.082\right)$, coordination and upper limb speed $\left(\mathrm{F}_{3,102}=8.19 \mathrm{P}>0.001, \mathrm{\eta}^{2}=0.19\right)$. Regarding hand evaluation, there was not a functional improvement after interventions. The analysis of group interaction showed significant difference between groups in coordination and upper limb speed $\left(\mathrm{F}_{3,102}=5.21, \quad \mathrm{P}=0.002, \quad \eta^{2}=0.13\right)$, with improvement in the group that was submitted only to physical therapy after the third month of intervention $(\mathrm{P}=0.008)$.

The evaluation of muscle tonus through the modified Ashworth scale revealed a significant tonus decrease in elbow flexors $\left(\mathrm{X}_{2}=8.312, \mathrm{P}=0.040\right)$ and wrist flexors $\left(\mathrm{X}_{2}=7.839, \mathrm{P}=0.049\right)$ in btx-A group.

\section{Gait function evaluation}

When analyzing function and gait speed, we observed that in both tests, TUG $\left(F_{3,102}=6,5, \quad P>0.001, \quad \eta^{2}=0,16\right)$ and six minute walking test $\left(\mathrm{F}_{3,102}=13,36\right.$, $\mathrm{P}>0.001$ ), there was a significant improvement after the third month of interventions in both groups $(\mathrm{P}=0.02)$. However, there was not a difference between groups during follow-up.

\section{Discussion}

Our results suggest that there was an improvement in both groups, with no better results in the btx-A group for upper limb and gait function evaluation. In addition, physical therapy proved to be of greater value when considering improvement of upper limb functionality.

When analyzing the domains of FuglMeyer scale it was possible to demonstrate in both groups improvement of the global upper limb evaluation (category A), wrist stability (category B), coordination and speed (category D), six months after initial intervention. Both groups were submitted to a predetermined physical therapy protocol and showed similar results during the trial, with no superior performance in the btx-A patients. These results are similar to those observed by Meythaler et al. 6

Takekawa et al. showed that the association between btx-A and functional training improved functional skills in categories A, $\mathrm{B}$ and D of Fugl-Meyer scale from the third month of intervention. However, they also showed that coordination and movement speed are linked to functional improvement of other body segments, and that treatment maintenance is essential to normal movements regain. ${ }^{7}$

Wolf et al. suggested that btx-A injections combined with physical therapy in post-stroke patients lead to similar improve- ment of upper limb function when compared to placebo and physical therapy. ${ }^{8}$ Meythaler et al. compared btx-A associated with physical therapy and control groups, also showing a little impact in upper limb function when analyzing ADL. They explained the results achieved to a specific physical exercise program that involved lower limbs segments muscles not dependent on btx-A application. ${ }^{6}$

When analyzing paretic hand (category C), there was no functional improvement during trial protocol, regardless the allocation group. Wolf et al. showed that after a long treatment with btx-A injections and physical therapy there was a global improvement in paretic hand function, however, no effects were found when analyzing strength and physic function. ${ }^{8}$ In chronic post-stroke patients, Takekawa et al. demonstrated that after six months of treatment with btx-A and muscle training, there was no functional improvement in paretic hands, ${ }^{7}$ reinforcing that functional exercises to improve motor function in paretic hand of post-stroke patients requires a longer rehabilitation process, depending also of lesion extension. They suggested that mus-

Table 1. Demographic characteristics.

\begin{tabular}{|c|c|c|}
\hline & Control group $(n=20)$ & Treatment group $(\mathrm{n}=20)$ \\
\hline Age (years), Mean \pm SD & $52.05 \pm 12.51$ & $52.5 \pm 11.01$ \\
\hline Stroke time (months), Mean \pm SD & $32.05 \pm 14.89$ & $34.15 \pm 21.43$ \\
\hline $\begin{array}{l}\text { Gender, } \mathrm{n}(\%) \\
\text { Male } \\
\text { Female }\end{array}$ & $\begin{array}{c}12(60) \\
8(40)\end{array}$ & $\begin{array}{c}12(60) \\
8(40)\end{array}$ \\
\hline $\begin{array}{l}\text { Type of stroke, } n(\%) \\
\text { Ischemic } \\
\text { Hemorrhagic }\end{array}$ & $\begin{array}{c}19(95) \\
1(5)\end{array}$ & $\begin{array}{c}16(80) \\
4(20)\end{array}$ \\
\hline $\begin{array}{l}\text { Dominant hand before stroke, } \mathrm{n}(\% \\
\text { Right } \\
\text { Left }\end{array}$ & $\begin{array}{c}19(95) \\
1(5)\end{array}$ & $\begin{array}{c}16(80) \\
4(20)\end{array}$ \\
\hline $\begin{array}{l}\text { Side injured by stroke, n (\%) } \\
\text { Right } \\
\text { Left }\end{array}$ & $\begin{array}{c}13(65) \\
7(35)\end{array}$ & $\begin{array}{c}9(45) \\
11(55)\end{array}$ \\
\hline $\begin{array}{l}\text { Diseases before stroke, n (\%) } \\
\text { Hypertension } \\
\text { Diabetes Mellitus } \\
\text { Others }\end{array}$ & $\begin{array}{l}17(85) \\
4(20) \\
1(5)\end{array}$ & $\begin{array}{l}18(90) \\
4(20) \\
2(10)\end{array}$ \\
\hline
\end{tabular}

Table 2. Mean and standard deviation of the measurement of Modified Ashworth Scale (MAS) during the treatment period with btx-a and placebo.

\begin{tabular}{lccccc} 
Type/local application & Baseline & $\begin{array}{c}\text { MAS } \\
(3 \text { months })\end{array}$ & $\begin{array}{c}\text { MAS } \\
(6 \text { months })\end{array}$ & $\begin{array}{c}\text { MAS } \\
(9 \text { months })\end{array}$ & P \\
Btx-a Elbow $(\mathrm{n}=10)$ & $2.4( \pm 0.52)$ & $1.8( \pm 1.13)$ & $1.6( \pm 1.06)$ & $1.9( \pm 0.94)$ & 0.04 \\
Placebo Elbow $(\mathrm{n}=11)$ & $2.4( \pm 0.53)$ & $1.7( \pm 0.87)$ & $1.9( \pm 0.88)$ & $1.8( \pm 0.93)$ & 0.184 \\
\hline Btx-a Wrist $(\mathrm{n}=5)$ & $2( \pm 0.0)$ & $0.8( \pm 1.09)$ & $0.7( \pm 0.97)$ & $0.9( \pm 0.89)$ & 0.049 \\
Placebo Wrist $(\mathrm{n}=4)$ & $2( \pm 0.0)$ & $1.3( \pm 1.15)$ & $2( \pm 0.0)$ & $2( \pm 0.0)$ & 0.392 \\
\hline
\end{tabular}


cle training programs are essential to stimulate the sustained use of the paretic hand with positive consequences in motor response. $^{7}$

Our results also demonstrated an improvement in gait as assessed by TUG and six minutes walking test in both allocation groups. These results reinforce the theory that physical therapy alone may be responsible for global motor function improvement in post-stroke patients. Btx-a injections would function as an adjuvant therapy, adding the fact that positive results were observed in segments with no btx-A injections.

Even with no differences in evolution of functional recovery of upper limb between groups, there was a significant reduction in muscle spasticity among patients who used btx-A injections in upper limb, as expected by btx-A mechanism of action. Upper limb spasticity may interfere with self-care activities as hand opening, dressing or drinking a glass of water. , $9-12^{-1 n}$ this way, several studies demonstrate reduction in upper limb muscles spasticity after btx-A injections. ${ }^{8,12-14}$ In a recent meta-analysis there was evidence that btx-A injections improve upper limb functionality. ${ }^{4}$ Meythaler et al. showed spasticity reduction in elbow and wrist extension after six months of btx-A injections. Nevertheless, patients submitted only to a physical therapy program showed similar results. ${ }^{6}$ Significant reduction of spasticity was also observed after six weeks of inducted contention therapy. 15

The current study has some limitations. First, there was variability in functional capacity for upper limb between study participants, with potential for bias. Also, the absence of data on ADL after study protocol impaired a subjective evaluation and comparison with objective results obtained through scales used in the study. Besides the restrict training program, there was not possible to control participants number of absences during trial scheduled visits.

\section{Conclusions}

The results showed that isolated physiotherapy as their association with btx-a improved motor function post-stroke individuals, without superiority among the techniques. However, the association of btx-a with physical therapy was higher in the control of muscle tone, suggesting that the functional improvement depends not only on decreased muscle tone.

\section{References}

1. Aguilar-Barberá M, Bori-Fortuny I, García-Aymerich V, et al. Guía terapéutica de la espasticidad del adulto con toxina botulínica. Rev Neurol 2004; 38:971-8.

2. Bhimani R, Anderson L. Clínical Understanding of Spasticity: Implications for Practice. Rehabil Res Pract 2014;2014:1-10.

3. Lundstrom E, Terent A, Borg J. Prevalence of disabling spasticity 1 year after first-ever stroke. Eur J Neurol 2008;15:533-9.

4. Foley N, Pereira S, Salter K, et al. Treatment with Botulinum Toxin improves upper-extremity function post Stroke: a systematic review and MetaAnalysis. Arch Phys Med Rehabil 2013;94:977-89.

5. You Y, Her JG, Woo JH, et al. The effects of stretching and stabilization exercise on the improvement of spastic shoulder function in hemiplegic patients. J Phys Ther Sci 2014;26:4915.

6. Meythaler JM, Vogtle L, Brunner RC. A preliminary assessment of the benefits of the addition of botulinum toxin $\mathrm{A}$ to a conventional therapy program on the function of people with longstanding stroke. Arch Phys Med Rehabil 2009;90:1453-61.

7. Takekawa T, Abo M, Ebihara K et al. Long-term effects of injection of botulinum toxin type A combined with home- based functional training for post-stroke patients with spastic upper limb hemiparesis. Acta Neurol Belg 2013;113:469-75.

8. Wolf SL, Milton B, Reiss A, et al. Further assessment to determine the additive effect of botulinum toxin type A on an upper extremity exercise program to enhance function among individuals with chronic stroke but extensor capability. Arch Phys Med Rehabil 2012;93:578-87.

9. Hesse S, Werner C. Poststroke motor dysfunction and spasticity: novel pharmacological and physical treatment strategies. CNS Drugs 2003;17:1093107.

10. Brashear A, Gordon MF, Elovic E, et al. Intramuscular injection of botulinum toxin for the treatment of wrist and finger spasticity after a stroke. N Engl J Med 2002;347:395-400.

11. Elovic EP, Brashear A, Kaelin D, et al. Repeated treatments with botulinum toxin type a produce sustained decreases in the limitations associated with focal upper-limb poststroke spasticity for caregivers and patients. Arch Phys Med Rehabil 2008;89:799-806.

12. Shackley P, Shaw L, Price C, et al. Cost-effectiveness of treating upper limp spasticity due to stroke with botulinum toxin type A: Results from the botulinum toxin for the upper limb after stroke (BoTULS) trial. Toxins 2012;4:1415-26.

13. Chang CL, Munin MC, Skidmore ER, et al. Effect of baseline spastic hemiparesis on recovery of upper-limb function following botulinum toxin type A injections and postinjection therapy. Arch Phys Med Rehabil 2009;90:14628.

14. Lin, Y. Botulinum toxin injection for Post-stroke spasticity. Muscle Nerve 2014;49:932.

15. Kagawa S, Koyama T, Hosomi M, et al. Effects of constraint-induced movement therapy on spasticity in patients with hemiparesis after stroke. J Stroke Cerebrovasc Dis 2013;22:364-70. 